\title{
Generating functions and sum rules for quantum oscillator
}

\author{
V. S. Popov and M. A. Trusov* \\ ITEP, Moscow, Russia
}

(Dated: March 23, 2009)

\begin{abstract}
Generating functions and sum rules are discussed for transition probabilities between quantum oscillator eigenstates with time-dependent parameters.
\end{abstract}

PACS numbers: 03.65.-w, 03.65.Fd

Keywords: Disentangling; Sum rules; Generating functions

The approach supposed by Feynman [1] for disentangling of non-commuting operators (FDM) was successfully applied to various time-dependent problems in quantum mechanics [2]-[5]. Using FDM one can [1, 3] calculate the transition probabilities between eigenstates $|m, t \rightarrow-\infty\rangle$ and $|n, t \rightarrow+\infty\rangle$ of a quantum oscillator with definite initial and final quantum numbers, and compute corresponding generating functions. If the oscillator has a constant frequency $\omega$ being under an external force $f(t)^{1}$, for the generating function one has ${ }^{2}$ :

$$
G(u, v \mid \nu)=\sum_{m, n=0}^{\infty} w_{m n}(\nu) u^{m} v^{n}=(1-u v)^{-1} \exp \left\{-\nu \frac{(1-u)(1-v)}{1-u v}\right\}
$$

where $u, v$ are the auxiliary complex numbers and $\nu$ is the oscillator excitation parameter:

$$
\nu=\frac{1}{2 \omega}\left|\int_{-\infty}^{\infty} f(t) \exp (-i \omega t) d t\right|^{2}, \quad \hbar=m=1 .
$$

From (11) one obtains some non-trivial relations for probabilities $w_{m n}$. First, integrating by $\nu$, one can derive several peculiar sum rules

$$
\begin{aligned}
& \int_{0}^{\infty} w_{m n}(\nu) d \nu=1, \quad\langle\nu\rangle_{m n}=\int_{0}^{\infty} w_{m n}(\nu) \nu d \nu=m+n+1, \\
& \left\langle\Delta \nu^{2}\right\rangle_{m n}=\int_{0}^{\infty} w_{m n}(\nu)\left[\nu-\langle\nu\rangle_{m n}\right]^{2} d \nu=2 m n+m+n+1
\end{aligned}
$$

for arbitrary quantum numbers $m$ and $n$. For the initial vacuum state $(m=0)$ we have $\left\langle\Delta \nu^{2}\right\rangle_{0 n}=\langle\nu\rangle_{0 n}$ which corresponds to the Poisson distribution.

Taking in (11) $u=v$ one has

$$
G(u, u \mid \nu)=\left(1-u^{2}\right)^{-1} \exp \left\{2 \nu u(1+u)^{-1}-\nu\right\}
$$

which yields

$$
S_{k}(\nu) \equiv \sum_{m+n=k} w_{m n}(\nu)=e^{-\nu} p_{k}(\nu)
$$

where $p_{k}(\nu)=\sum_{s=0}^{k}(-1)^{s} L_{s}(2 \nu)$ and $L_{s}$ are the Laguerre polynomials, so

$$
p_{0}=1, \quad p_{1}=2 \nu, \quad p_{2}=2 \nu^{2}-2 \nu+1, \quad p_{3}=\frac{4}{3} \nu^{3}-4 \nu^{2}+4 \nu, \ldots
$$

\footnotetext{
*Electronic address: trusov@itep.ru

1 A natural condition $f(t) \rightarrow 0$ at $t \rightarrow \pm \infty$ is supposed.

2 This expression can be easily derived [5] from Schwinger result [6] for a sum $\sum_{m=0}^{\infty} w_{m n} u^{m}$.
} 
For the oscillator with variable frequency $\omega(t)$ without an external force ${ }^{3}(f(t) \equiv 0)$ one obtains

$$
G(u, v \mid \rho)=\sum_{m, n=0}^{\infty} w_{m n}(\rho) u^{m} v^{n}=\sqrt{(1-\rho) /\left[(1-u v)^{2}-\rho(u-v)^{2}\right]}
$$

and

$$
\int_{0}^{1} G(u, v \mid \rho)(1-\rho)^{-1} d \rho=\frac{2}{u-v}(\operatorname{Arctanh} u-\operatorname{Arctanh} v) .
$$

Here $\rho, 0 \leq \rho \leq 1$ is the oscillator excitation parameter; details can be found in [3, $[5]$. The frequency $\omega(t)$ is an arbitrary real time function. As usual, we propose the boundary conditions:

$$
\omega(t) \rightarrow \omega_{ \pm} \quad \text { at } \quad t \rightarrow \pm \infty
$$

which allows one to define the final and initial eigenstates of the oscillator. Note that the expressions (1) and (5) result from the general Husimi expression [7].

As a result we have

$$
\begin{gathered}
\int_{0}^{1} \frac{w_{m n}(\rho)}{1-\rho} d \rho=\frac{1+(-1)^{m+n}}{m+n+1}, \\
\int_{0}^{1} \frac{w_{m n}(\rho)}{\rho \sqrt{1-\rho}} d \rho=\frac{1+(-1)^{m+n}}{|m-n|}, \quad m \neq n .
\end{gathered}
$$

Analogously to (3) let us calculate the integral $J_{m n}=\int_{0}^{1} w_{m n}(\rho) d \rho$. For diagonal $(m=n)$ transitions

$$
J_{n n}=\frac{1}{2 n+1}\left[1+\frac{1}{(2 n+3)(2 n-1)}\right], \quad n=0,1,2, \ldots,
$$

at $m \neq n$ the expression for $J_{m n}$ is more cumbersome.

Finally, taking in (5) $u=v$, one obtains

$$
G(u, u \mid \rho)=\frac{\sqrt{1-\rho}}{1-u^{2}}
$$

and

$$
S_{k}(\rho)=\sum_{m+n=k} w_{m n}(\rho)= \begin{cases}\sqrt{1-\rho}, & k=0,2,4, \ldots \\ 0, & k=1,3,5, \ldots\end{cases}
$$

(compare to (4)).

On differentiating subsequently the generating functions on parameters $u$ and $v$, one can calculate the average quantum number in the final state

$$
\left.\frac{\partial G}{\partial v}\right|_{v=1}=\sum_{m=0}^{\infty}\langle n\rangle_{m} u^{m}, \quad\langle n\rangle_{m}=\sum_{n=0}^{\infty} n w_{m n}=-\frac{1}{2}+\left(m+\frac{1}{2}\right) \frac{1+\rho}{1-\rho}
$$

the dispersion $\left\langle\Delta n^{2}\right\rangle_{m}$ and other higher distribution momenta.

\footnotetext{
${ }^{3}$ For this case the transitions occur only between states with same parity.
} 
In [4] a more general model of a singular oscillator with variable frequency was considered:

$$
\begin{gathered}
\hat{H}=\frac{1}{2} p^{2}+\frac{1}{2} \omega(t)^{2} x^{2}+\frac{g}{8 x^{2}}, \\
0<x<+\infty, \quad g=\text { const }, \quad g>-1 .
\end{gathered}
$$

It is well known that at a fixed $t$ the instantaneous spectrum of the Hamiltonian (11) is equidistant (see, e.g., [8]):

$$
E_{n}=2 \omega(n-j), \quad j=-\frac{1}{2}-\frac{1}{4} \sqrt{1+g}, \quad n=0,1,2, \ldots
$$

Using FDM, one can disentangle the operators in $H$ and, after application of some group theory methods, calculate the transition amplitudes between initial $|m\rangle$ and final $|n\rangle$ states, being expressed in terms of the generalized Wigner function for the irreducible representation of the $s u(1,1)$ algebra with weight $j$. As a result, one obtains explicit analytical expressions for the transition probabilities $w_{m n}$. The corresponding generating function takes the form:

$$
g(u, v)=\sum_{m, n} w_{m n} u^{m} v^{n}=\frac{\lambda^{-2 j}}{1-u v \cdot \lambda^{2}}
$$

where

$$
\lambda=\frac{2(1-\rho)}{1-\rho(u+v)+u v+\sqrt{[1-\rho(u+v)+u v]^{2}-4 u v(1-\rho)^{2}}}
$$

(see [9] for details).

One can show that at $j=-3 / 4$, which corresponds to the regular oscillator $(g=0)$ with odd levels, these expressions turn into (5).

The most interesting physical case is the excitation of the oscillator ground level, i.e. $m=0$. Taking in (13) $u=0$ and, correspondingly, $\lambda=(1-\rho) /(1-\rho v)$, one obtains

$$
g(0, v)=\sum_{n=0}^{\infty} w_{0 n} v^{n}=\left(\frac{1-\rho}{1-\rho v}\right)^{-2 j}
$$

and

$$
w_{0 n}(\rho)=\frac{\Gamma(n-2 j)}{n ! \Gamma(-2 j)} \rho^{n}(1-\rho)^{-2 j}, \quad n=0,1,2, \ldots
$$

This formula also yields [3] the transition probabilities of the regular oscillator for $j=-1 / 4$ (even states, $0^{\text {th }}$ level excitation) and for $j=-3 / 4$ (odd states, $1^{\text {th }}$ level excitation).

To conclude, let us note that the generating function (13) determines also the adiabatic expansion of the probability $w_{m n}$ for the case of small values of the excitation parameter. For example, for diagonal transitions $(m=n)$ one has

$$
w_{n n}(\rho)=1-2\left[n^{2}-(2 n+1) j\right] \rho+\cdots=1-\frac{1}{2}\left(N^{2}+N+1\right) \rho+\mathcal{O}\left(\rho^{2}\right)
$$

where

$$
N=2 \sqrt{(n-j)^{2}-(j(j+1)+3 / 16)}-\frac{1}{2} .
$$

In particular, $N=2 n$ for $j=-1 / 4$ and $N=2 n+1$ for $j=-3 / 4$.

\section{Acknowledgments}

This work was partially supported by the Russian Foundation for Basic Research (grant No. 07-02-01116) and by the Ministry of Science and Education of the Russian Federation (grant No. RNP 2.1.1. 1972). One of the authors (M.A.T.) also thanks for partial support the President Grant No. NSh-4961.2008.2 and the President Grant No. MK-2130.2008.2.

[1] R. P. Feynman, Phys. Rev. 84, 108 (1951). 
[2] V. S. Popov, Zh. Eksp. Teor. Fiz. 35, 985 (1958).

[3] V. S. Popov, Phys. Lett. A342, 281 (2005).

[4] V. S. Popov and M. A. Trusov, Phys. Lett. A372, 5274 (2008).

[5] V. S. Popov, Phys. Usp. 50, 1217 (2007).

[6] J. Schwinger, J. Math. Phys. 2, 407 (1961).

[7] K. Husimi, Prog. Theor. Phys. 9, 381 (1953).

[8] L. D. Landau and E. M. Lifshitz, Quantum Mechanics: Non-Relativistic Theory (Pergamon Press, 1977).

[9] M. A. Trusov, in preparation. 